\title{
Dietary effects on fatty acid composition in muscle tissue of juvenile European eel, Anguilla anguilla (L.)
}

\author{
Enno Prigge $\cdot$ Arne M. Malzahn • \\ Karsten Zumholz $\cdot$ Reinhold Hanel
}

Received: 18 May 2010 / Revised: 19 January 2011 / Accepted: 21 January 2011 / Published online: 8 February 2011

(C) Springer-Verlag and AWI 2011

\begin{abstract}
The role of intracontinental migration patterns of European eel (Anguilla anguilla) receives more and more recognition in both ecological studies of the European eel and possible management measures, but small-scale patterns proved to be challenging to study. We experimentally investigated the suitability of fatty acid trophic markers to elucidate the utilization of feeding habitats. Eight groups of juvenile European eels were fed on eight different diets in a freshwater recirculation system at $20^{\circ} \mathrm{C}$ for 56 days. Three groups were fed on freshwater diets (Rutilus rutilus, Chironomidae larvae, and Gammarus pulex) and four groups were reared on diets of a marine origin (Clupea
\end{abstract}

Communicated by H.-D. Franke.

E. Prigge $(\square) \cdot$ K. Zumholz

Leibniz-Institute of Marine Sciences, IFM-GEOMAR,

Düsternbrooker Weg 20, 24105 Kiel, Germany

e-mail: eprigge@ifm-geomar.de

\section{A. M. Malzahn}

Alfred-Wegener-Institut für Polar- und Meeresforschung,

Biologische Anstalt Helgoland, Postbox 180,

27483 Helgoland, Germany

A. M. Malzahn

Helmholtz-Zentrum Geesthacht,

Centre for Materials and Coastal Research,

Institute for Coastal Research, Max-Planck-Straße 1,

21502 Geesthacht, Germany

R. Hanel

Johann Heinrich von Thünen-Institut,

Federal Research Institute for Rural Areas, Forestry and Fisheries, Institute for Fisheries Ecology, Palmaille 9,

22767 Hamburg, Germany

K. Zumholz

Berufsbildungszentrum am Nord-Ostseekanal, Fischereischule, Am Kamp 13, 24768 Rendsburg, Germany harengus, Crangon crangon, Mysis spec., and Euphausia superba) and one on commercial pellets used in eel aquaculture. Fatty acid composition (FAC) of diets differed significantly with habitat. FAC of eel muscle tissue seemed to be rather insensitive to fatty acids supplied with diet, but the general pattern of lower $n 3: n 6$ and EPA:ARA ratios in freshwater prey organisms could be traced in the respective eels. Multivariate statistics of the fatty acid composition of the eels resulted in two distinct groups representing freshwater and marine treatments. Results further indicate the capability of selectively restraining certain fatty acids in eel, as e.g. the n3:n6 ratio in all treatments was $<4$, regardless of dietary n3:n6. In future studies on wild eel, these measures can be used to elucidate the utilization of feeding habitats of individual European eel.

Keywords Catadromy - Habitat utilization - Freshwater . Marine $\cdot$ Salinity $\cdot$ Fatty acids $\cdot$ Trophic ecology

\section{Introduction}

Despite the relatively small number of datasets that provide information on the recruitment of the European eel (Anguilla anguilla, L.), it is widely accepted that both recruitment and stock size of the species have been in a dramatic downward trend for the last two decades (ICES 2008). Today, the stock is considered to be out of safe biological limits and A. anguilla has been listed in the red book of endangered species all over Europe (Dekker 2003; ICES 2008). As a result of numerous (most likely interacting) factors, estimates of actual spawning stock biomass have been reduced to $2-12 \%$ and the abundance of glass eels to less than $5 \%$ of its level prior to the early 1980s (ICES 2002, 2006; Bonhommeau et al. 2008). 
It has been widely speculated that following metamorphosis from glass eel stage, juvenile eels migrate into continental freshwaters (Tesch 1999). However, it is still under discussion to which extent yellow eels move between freshwater and marine habitats during their continental phase (Daverat et al. 2006; Shiao et al. 2006; Thibault et al. 2007a; Thibault et al. 2007b). In order to substantially monitor and protect the European eel stock, it is therefore essential to gain better knowledge about the whereabouts of the stock and/or individual eels in continental waters and to fill up existing gaps in the knowledge of its biology.

The feeding habits of the European eel are generally accepted to be extraordinarily diverse and variable (Tesch 1999), and the European eel is often considered to show great trophic opportunism (Sinha et al. 1975; Tesch 1999; Schulze et al. 2004). Such conclusions are usually drawn from stomach content analysis, which are known to harbor a number of biases both in sampling and interpretation (Cortes 1997) and can hardly reveal more than very recent feeding due to variable digestion and gut evacuation. It is therefore desirable to supplement knowledge of feeding and foraging ecology based on stomach samples with an additional time-integrating biochemical method e.g. analysis of consumer stable isotope ratios or fatty acids (Kuusipalo and Kakela 2000; Harrod et al. 2005; Vinson and Budy 2011).

As most fish are incapable of synthesizing polyunsaturated fatty acids (PUFA) de novo, they are completely dependent on dietary uptake of these nutrients. Therefore, fatty acid compositions (FAC) of higher trophic levels reflect the availability of fatty acid (FA) in the food chain (Henderson and Tocher 1987). Numerous studies have documented various influences of diet on the FAC in fish (Boggio et al. 1985; Saito et al. 1997; Steffens 1997; Kirsch et al. 1998; Saito and Murata 1998; Saito et al. 2002). As FAC reflects the integration of food items over weeks to months, it might therefore be more convincing for drawing general conclusions than stomach samples (Arim and Naya 2003). Fatty acids are potentially suitable for the use as biomarkers for feeding and/or foraging behavior. This is due to their biological specificity and the fact that many fatty acids are transferred conservatively through food webs from primary producers to succeeding trophic levels. On strength of those characteristics distinct differences in, e.g. n3:n6 and the eicosapentaenoic acid to arachidonic acid (EPA:ARA) ratios can be found between freshwater and marine organisms (Lovern 1935; Reiser et al. 1963; D’Abramo 1979).

The objective of the present study was to examine the role of dietary influence on the fatty acid composition in European eel provided with foods of known and contrasting FACs. Furthermore, the study aimed to examine the potential of fatty acid analysis (FAA) to determine the habitat of wild-caught animals. Different potential marine and freshwater food sources were provided to juvenile European eel (Anguilla anguilla) in a controlled laboratory experiment. Subsequent to the feeding period, individual fatty acid profiles were examined in muscle tissue to trace back dietary influence.

\section{Materials and methods}

Animal husbandry and experimental design

Pigmented juvenile eels (Anguilla anguilla) were obtained from a commercial fish farm (Fischzucht Reese; Sarlhusen, Germany) in May 2008. Acclimatization to water conditions was ensured by keeping the eels in a 500-liter freshwater tank for 8 weeks at $\sim 20^{\circ} \mathrm{C}$. The eels were fed with commercial pellets (A 0.7 Perle Eel; Skretting) every second day. The water was renewed once a week. For the feeding trial (56 days), 96 eels were randomly taken out of the acclimatization tank and transferred into a freshwater recirculation system in a temperature-controlled room at $20^{\circ} \mathrm{C}$ with a $12 \mathrm{~h} / 12 \mathrm{~h}$ light regime. Eels selected for the experiment had a mean $( \pm \mathrm{SD})$ total length of $18.8 \pm$ $1.6 \mathrm{~cm}$ and mean $( \pm \mathrm{SD})$ mass of $8.4 \pm 2.3 \mathrm{~g}$. The recirculation system consisted of 32 plastic tanks $(360 \times 193 \times$ $208 \mathrm{~mm}$ ) with in- and outflow installed to achieve a filling volume of approximately $8 \mathrm{~L}$. Water exchange in the tanks was adjusted to be threefold to fourfold per day. The tanks were connected to a biofilter with a total volume of $60 \mathrm{~L}$ and a bioactive surface area of $\sim 23 \mathrm{~m}^{2}$. Water lost by evaporation in the biofilter was balanced with freshwater every day. Plastic tubes were provided as shelter. To prevent the eels from escaping, plastic covers were mounted on top of each tank. The eels were divided into 32 groups of three individuals each. The eight different diets were allocated to the 32 tanks, resulting in four replicates per treatment. In order to document individual growth, visible implant elastomer tags (Northwest Marine Technology, Inc., Shaw Island, WA, U.S.A.) were applied to each eel resulting in an individual code.

\section{Experimental diets}

With respect to the facultative catadromous lifecycle and the related broad range of possible food items of the European eel, putative fresh-, brackish-, and saltwater organisms were chosen as experimental diets. Diets were kept frozen at $-20^{\circ} \mathrm{C}$. Prior to feeding, food organisms were defrosted and fed ad libitum in bite-sized pieces or as whole organisms once a day. Leftover food was removed every day prior to feeding.

Roach (Rutilus rutilus) was chosen to represent a freshwater piscivorous diet. $R$. rutilus was caught in a freshwater 
pond (Dörpsee, Emkendorf) near Kiel, Germany. Small pieces (approximately $0.5 \mathrm{~cm}^{3}$ ) of filet were fed. As gammarids are considered to contribute a major part of small eel diet in freshwater habitats (Mann and Blackburn 1991), Gammarus pulex was chosen to represent a freshwater crustacean diet. G. pulex was caught in the freshwater River Eider (Kiel, Germany) and fed as whole animals. Representing a possible insect diet of the European eel, Chironomidae larvae were obtained frozen (Claudia Erdmann $\mathrm{GmbH}$, Germany) and fed as whole individuals. For logistic reasons, herring (Clupea harengus) was chosen as potential marine piscivorous diet of European eel, even though eels are unlikely to feed on herring in the wild. C. harengus was obtained frozen from a commercial fishery (Wiese Eduard \& Kruse Ivens GmbH, Germany). Small pieces of dorsal muscle (approximately $0.5 \mathrm{~cm}^{3}$, skin included) were fed to the eels. To represent marine crustacean diets, we used Pacific krill Euphausia superba and mysids. Both were supplied frozen by an aquarium food manufacturer (Claudia Erdmann GmbH, Germany) and fed as whole organisms. To account for possible feeding on brackish water crustaceans, the brown shrimp (Crangon crangon) was a representative for crustacean diet. C. crangon was caught in Kiel Bight and with respect to the hydrological properties of the Western Baltic should be considered a brackish water diet. However, for statistical analysis, it was assigned to "marine diets." Small pieces (approximately $0.5 \mathrm{~cm}^{3}$ ) were fed. Commercial pellets (A 0.7 Perle Eel) were obtained from a fish feed producer (Skretting) and stored at $4^{\circ} \mathrm{C}$.

Sample analysis

At the end of the feeding period (56 days), the eels were anaesthetized with ethylene glycol monophenyl ether $(5 \mathrm{ml} /$ $101 ; 0.0005 \%$ aqueous solution), killed, and frozen at $-80^{\circ} \mathrm{C}$. Cross-sectional samples of the same position and portion (approximately $0.5 \mathrm{~cm}$, anterior of the dorsal fin) were taken from each animal. Approximately 200 to $300 \mu \mathrm{g}$ of dorsal muscle tissue was extracted from each sample. Lipid extraction was conducted by storing the samples in $2 \mathrm{ml}$ Dichlormethan/Methanol $(2: 1)$ at $-80^{\circ} \mathrm{C}$ for 4 days. The FAC of eels and diets was analyzed as fatty acid methyl esters (FAMEs) after Malzahn et al. (2007). Resulting chromatograms were postprocessed using GALAXIE CHROMATOGRAPHY WORKSTATION (Version 1.7; Varian Inc.).

\section{Statistical analysis}

All statistics were done in STATISTICA 8.0 (StatSoft). Since FAC was measured as percentage of total FA content, the dataset was arcsin-transformed for statistical analysis. Specific growth rate was checked for significant differences and tank effects with nested ANOVA with "Diet" and "Tank" assigned as factors. "Tank" was treated as random factor and nested in "Diet." In order to document distinction of diets and eels by their FAC, two separate analyses were conducted. (I) To confirm diet-habitat, diet samples were checked for significant differences in fatty acid concentrations and fatty acid ratios previously described to differ between habitats (marine and freshwater) by analysis of variance followed by post hoc comparison of means with Fisher LSD test. (II) To distinguish the eels by means of their FAC, first, a principle component analysis (PCA) followed by a factor analysis was conducted. Subsequently, nested ANOVA was used to check for significant differences in fatty acids and to exclude tank effects. "Diet" and "Tank" were assigned as factors with "Tank" treated as random factor and nested in "Diet." To assess the differences between treatments, post hoc comparison of means using Fisher LSD test was performed. In all tests, level of significance was set to be 0.05 .

The analysis of FACs as a whole required a multivariate statistical approach. Therefore, multidimensional scaling (MDS) based on Bray-Curtis similarity coefficient was used (PRIMER Version 6; PRIMER-E). Statistical differences in total FAC between diets and eels fed on freshwater and marine diets, respectively, were determined using ANOSIM (PRIMER Version 6; PRIMER-E).

\section{Results}

Growth performance

Initial weight did not differ significantly between treatments (ANOVA, $P>0.05$ ). Eels accepted all diets, albeit some diets resulted in poor growth. Negative weight development in the "Gammarus pulex" and "Euphausia superba" groups was reflected in negative SGRs of $-0.11 \pm 0.07 \% \mathrm{Wday}^{-1}$

Table 1 Growth performance of eels at different treatments

\begin{tabular}{llrr}
\hline Diet & $\begin{array}{l}\text { Avg } \\
\text { inital wt [g] }\end{array}$ & \multicolumn{1}{l}{$\begin{array}{l}\text { Avg } \\
\text { final wt [g] }\end{array}$} & \multicolumn{1}{l}{$\begin{array}{l}\text { SGR } \\
{\left[\% \mathrm{~d}^{-1}\right]}\end{array}$} \\
\hline Rutilus rutilus & $8.3 \pm 0.4$ & $9.9 \pm 0.8$ & $0.30 \pm 0.07$ \\
Chironomidae larvae & $8.7 \pm 0.7$ & $9.3 \pm 1.1$ & $0.06 \pm 0.08$ \\
Gammarus pulex & $7.1 \pm 0.3$ & $6.7 \pm 0.6$ & $-0.11 \pm 0.07$ \\
Pellets & $8.5 \pm 0.8$ & $9.5 \pm 0.9$ & $0.22 \pm 0.11$ \\
Clupea herangus & $9.2 \pm 0.8$ & $11.1 \pm 1.0$ & $0.33 \pm 0.10$ \\
Crangon crangon & $7.3 \pm 0.3$ & $8.6 \pm 0.4$ & $0.29 \pm 0.08$ \\
Mysis spec & $9.8 \pm 0.9$ & $10.4 \pm 0.9$ & $0.13 \pm 0.08$ \\
Euphausia superba & $8.3 \pm 0.6$ & $7.1 \pm 0.4$ & $-0.25 \pm 0.05$ \\
\hline
\end{tabular}

$\overline{W t}=$ wet weight; $S G R=$ specific growth rate $\left(\%\right.$ weight day $\left.{ }^{-1}\right)$; all values given $\pm \mathrm{SE}$ 
Table 2 Significant differences in growth performance

\begin{tabular}{|c|c|c|c|c|c|c|c|c|}
\hline & SGR $\left[\% \mathrm{~d}^{-1}\right]$ & Rutilus rutilus & $\begin{array}{l}\text { Chironomidae } \\
\text { larvae }\end{array}$ & $\begin{array}{l}\text { Gammarus } \\
\text { pulex }\end{array}$ & Pellets & $\begin{array}{l}\text { Clupea } \\
\text { herangus }\end{array}$ & $\begin{array}{l}\text { Crangon } \\
\text { crangon }\end{array}$ & Mysis spec \\
\hline Rutilus rutilus & $(0.30)$ & & & & & & & \\
\hline Chironomidae larvae & $(0.03)$ & 0.04 & & & & & & \\
\hline Gammarus pulex & $(-0.11)$ & 0.00 & 0.16 & & & & & \\
\hline Pellets & $(0.22)$ & 0.53 & 0.16 & 0.00 & & & & \\
\hline Clupea herangus & $(0.33)$ & 0.81 & $\mathbf{0 . 0 3}$ & 0.00 & 0.39 & & & \\
\hline Crangon crangon & $(0.29)$ & 0.96 & 0.05 & 0.00 & 0.57 & 0.77 & & \\
\hline Mysis spec & $(0.13)$ & 0.15 & 0.56 & 0.5 & 0.41 & 0.09 & 0.16 & \\
\hline Euphausia superba & $(-0.25)$ & 0.00 & 0.01 & 0.23 & 0.00 & 0.00 & 0.00 & 0.00 \\
\hline
\end{tabular}

Labels indicate organisms fed to the eels. Significant differences are highlighted

Values in brackets are SGR $\left(\% \mathrm{~d}^{-1}\right)$

Table 3 Main effects of "Diet" or "Diet-Habitat" and "Tank" determined by nested analysis of variance (general linear model, type III decomposition)

\begin{tabular}{|c|c|c|c|c|c|}
\hline Variable & Factor & SS* & $\mathrm{df}$ & $\mathrm{F}$ & $P$ \\
\hline \multirow[t]{3}{*}{ SGR** } & Tank (Diet) & 1.15405 & 24 & 0.497 & 0.97 \\
\hline & Diet & 3.67474 & 7 & 10.92 & $<0.01$ \\
\hline & Error & 6.19222 & 64 & & \\
\hline \multirow[t]{3}{*}{ 20:6 n3 (EPA) } & Tank (Diet-habitat) & 0.01809 & 30 & 0.83 & 0.69 \\
\hline & Diet-habitat & 0.01371 & 1 & 22.72 & $<0.01$ \\
\hline & Error & 0.04607 & 64 & & \\
\hline \multirow[t]{3}{*}{ 20:4 n6 (ARA) } & Tank (Diet-habitat) & 0.00353 & 30 & 1.04 & 0.44 \\
\hline & Diet-habitat & 0.00019 & 1 & 1.68 & 0.21 \\
\hline & Error & 0.00726 & 64 & & \\
\hline \multirow[t]{3}{*}{ 22:6 n3 (DHA) } & Tank (Diet-habitat) & 0.04785 & 30 & 1.16 & 0.30 \\
\hline & Diet-habitat & 0.01692 & 1 & 10.61 & $<0.01$ \\
\hline & Error & 0.087762 & 64 & & \\
\hline \multirow[t]{3}{*}{ EPA/ARA } & Tank (Diet-habitat) & 0.04523 & 30 & 1.05 & 0.42 \\
\hline & Diet-habitat & 0.02887 & 1 & 19.15 & $<0.01$ \\
\hline & Error & 0.09173 & 64 & & \\
\hline \multirow[t]{3}{*}{ n3 } & Tank (Diet-Habitat) & 0.07960 & 30 & 1.07 & 0.40 \\
\hline & Diet-Habitat & 0.02931 & 1 & 11.05 & $<0.01$ \\
\hline & Error & 0.15882 & 64 & & \\
\hline \multirow[t]{3}{*}{ n6 } & Tank (Diet-habitat) & 0.00837 & 30 & 1.48 & 0.10 \\
\hline & Diet-habitat & 0.00263 & 1 & 5.41 & $<0.01$ \\
\hline & Error & 0.01209 & 64 & & \\
\hline \multirow[t]{3}{*}{ n3/n6 } & Tank (Diet-habitat) & 0.01358 & 30 & 1.43 & 0.12 \\
\hline & Diet-habitat & 0.00784 & 1 & 17.32 & $<0.01$ \\
\hline & Error & 0.02031 & 64 & & \\
\hline
\end{tabular}

"Diet" or "Diet-Habitat" was treated as fixed factor, and "Tank" was treated as random factor. A significant effect is indicated by a $P$-value smaller 0.05

* Sum of squares

** Specific growth rate $\left[\% \mathrm{~d}^{-1}\right]$

for eels fed with G. pulex and $-0.25 \pm 0.05 \% \mathrm{Wday}^{-1}$ for eels fed with E. superba, respectively (Table 1). Growth performance differed significantly between treatments
$(P<0.05$; Table 2). Highest growth rates were documented in the two piscine treatments with $0.3 \pm 0.07 \% \mathrm{Wday}^{-1}$ for eels fed with $R$. rutilus and $0.33 \pm 0.1 \% \mathrm{Wday}^{-1}$ for eels fed with $C$. harengus (Table 1). No tank effects could be detected (Table 3).

Fatty acid composition

Postprocessing of the chromatograms resulted in the detection of a total of 30 fatty acids (Tables 4, 5). FAC of dietary organisms and eels was primarily dominated by the saturated fatty acids (SFA) C16:0 and C18:0, the monounsaturated fatty acids (MUFA) 18:1n-9, 16:1n-7, 22:1n-9 and 20:1n-9, and the polyunsaturated fatty acids (PUFA) 18:2n-6, 18:3n-3, 18:4n-3, 20:4n-6 (Arachidonic acid; ARA), 20:5n-3 (Eicosapentaenoic acid, EPA), 22:5n-3 and 22:6n-3 (Docosahexaenoic acid, DHA). All of these fatty acids were present to at least $2 \%$ of total FA content in more than one FAC of dietary organisms and/or eels (Tables 4,5 ). The remaining fatty acids accounted for only a minor part of the total FAC.

The most striking differences in FAC of prey organisms originating in freshwater and those from marine habitats were significantly lower n3:n6 (ANOVA, $P<0.05$ ) and EPA:ARA (ANOVA, $P<0.05$ ) ratios in freshwater diets than in marine diets (Table 3; Fig. 1). The pattern of a significantly lower n3:n6 ratio in freshwater diets was found again in eels (Table 3; Fig. 1).

Fatty acids and fatty acid ratios responsible for most of the variance in both diets and eels were identified by PCA (Table 6). Analysis of similarities showed a significant difference in FAC between diets fed to the eels (ANOSIM, $R=0.994, P=0.0001$, Fig. 2). Subsequently, analysis of similarities between those eels fed with a marine diet and those fed with a freshwater diet showed a significant difference in FAC (ANOSIM, $R=0.226, P=0.0001$, Fig. 2). Nevertheless, scattering within treatments was considerable. 
Table 4 Fatty acids ( $\%$ of total FAC \pm SD) of diets; "sat" = saturated fatty acids; "unsat" = unsaturated fatty acids; "PUFA" = poly unsaturated fatty acids; $\mathrm{a}=t$-test identified a significant difference $(P<0.05)$ between freshwater (Rutilus rutilus, Chironomidae larvae, and Gammarus pulex) and marine diets (Pellets, Clupea harengus, Mysis spec., and Euphausia superba)

\begin{tabular}{|c|c|c|c|c|c|c|c|c|}
\hline & $\begin{array}{l}\text { Rutilus rutilus } \\
(N=4)\end{array}$ & $\begin{array}{l}\text { Chironomidae } \\
\text { larvae }(N=4)\end{array}$ & $\begin{array}{l}\text { Gammarus } \\
\text { pulex }(N=4)\end{array}$ & $\begin{array}{l}\text { Pellets } \\
(N=4)\end{array}$ & $\begin{array}{l}\text { Clupea harengus } \\
(N=4)\end{array}$ & $\begin{array}{l}\text { Crangon } \\
\text { crangon }(N=4)\end{array}$ & $\begin{array}{l}\text { Mysis spec. } \\
(N=4)\end{array}$ & $\begin{array}{l}\text { Euphausia } \\
\text { superba }(N=4)\end{array}$ \\
\hline 16:0 & $29.36 \pm 3.40$ & $26.78 \pm 3.13$ & $18.97 \pm 1.87$ & $20.82 \pm 1.43$ & $20.72 \pm 4.03$ & $25.29 \pm 2.58$ & $21.70 \pm 1.72$ & $22.87 \pm 0.68$ \\
\hline $16: 1 \mathrm{n} 7$ & $1.84 \pm 0.96$ & $8.83 \pm 2.58$ & $2.94 \pm 1.39$ & $5.58 \pm 0.46$ & $2.82 \pm 1.11$ & $5.62 \pm 2.19$ & $5.13 \pm 0.56$ & $5.23 \pm 0.35$ \\
\hline $16: 2 \mathrm{n} 4$ & $0.48 \pm 0.29$ & $0.78 \pm 0.34$ & $0.64 \pm 0.14$ & $0.67 \pm 0.06$ & $0.21 \pm 0.12$ & $0.47 \pm 0.13$ & $0.29 \pm 0.03$ & $0.65 \pm 0.33$ \\
\hline $16: 3 \mathrm{n} 4$ & $0.11 \pm 0.23$ & $0.20 \pm 0.08$ & $0.33 \pm 0.05$ & $0.30 \pm 0.01$ & $0.25 \pm 0.07$ & $0.27 \pm 0.22$ & $0.07 \pm 0.01$ & $1.00 \pm 0.10$ \\
\hline 17:0 & $0.96 \pm 0.47$ & $1.79 \pm 0.20$ & $1.53 \pm 0.32$ & $1.53 \pm 0.03$ & $0.66 \pm 0.06$ & $1.44 \pm 0.18$ & $1.06 \pm 0.16$ & $2.07 \pm 0.12$ \\
\hline 17:1 n7 & $0.45 \pm 0.43$ & $0.95 \pm 0.24$ & $1.33 \pm 0.74$ & $1.09 \pm 0.09$ & $0.24 \pm 0.08$ & $0.83 \pm 0.50$ & $0.28 \pm 0.09$ & $0.85 \pm 0.13$ \\
\hline $18: 0^{\mathrm{a}}$ & $14.79 \pm 1.74$ & $11.39 \pm 0.82$ & $8.13 \pm 4.24$ & $6.20 \pm 0.84$ & $5.66 \pm 2.19$ & $8.83 \pm 5.46$ & $3.70 \pm 2.39$ & $2.82 \pm 0.29$ \\
\hline $18: 1 \mathrm{n} 9^{\mathrm{a}}$ & $18.07 \pm 2.92$ & $24.86 \pm 2.41$ & $30.30 \pm 2.98$ & $16.41 \pm 0.43$ & $11.25 \pm 2.33$ & $20.51 \pm 1.82$ & $12.87 \pm 1.22$ & $17.64 \pm 1.60$ \\
\hline 18:1 n7 & $0.15 \pm 0.05$ & $0.53 \pm 0.59$ & $0.42 \pm 0.13$ & $0.18 \pm 0.04$ & $0.23 \pm 0.06$ & $0.23 \pm 0.07$ & $0.48 \pm 0.04$ & $0.17 \pm 0.02$ \\
\hline $18: 2 n 6^{a}$ & $3.31 \pm 0.45$ & $13.15 \pm 2.32$ & $10.30 \pm 1.29$ & $4.96 \pm 0.46$ & $1.87 \pm 0.44$ & $1.79 \pm 0.32$ & $1.52 \pm 0.23$ & $2.08 \pm 0.09$ \\
\hline 18:3 n6 & $0.11 \pm 0.06$ & $0.21 \pm 0.09$ & $0.23 \pm 0.17$ & $0.21 \pm 0.02$ & $0.06 \pm 0.03$ & $0.11 \pm 0.05$ & $0.14 \pm 0.09$ & $0.20 \pm 0.01$ \\
\hline $18: 3 n 4^{a}$ & $0.29 \pm 0.09$ & $0.36 \pm 0.06$ & $0.44 \pm 0.15$ & $0.24 \pm 0.01$ & $0.10 \pm 0.02$ & $0.26 \pm 0.14$ & $0.14 \pm 0.03$ & $0.10 \pm 0.02$ \\
\hline $18: 3$ n3 & $2.14 \pm 0.97$ & $0.75 \pm 0.11$ & $5.27 \pm 2.36$ & $0.92 \pm 0.08$ & $0.74 \pm 0.32$ & $1.22 \pm 0.76$ & $0.91 \pm 0.17$ & $2.27 \pm 0.14$ \\
\hline $18: 4 n 3^{a}$ & $0.33 \pm 0.23$ & $0.10 \pm 0.02$ & $0.35 \pm 0.15$ & $2.03 \pm 0.16$ & $1.24 \pm 0.65$ & $0.16 \pm 0.05$ & $0.69 \pm 0.13$ & $4.71 \pm 0.50$ \\
\hline $20: 0^{\mathrm{a}}$ & $0.62 \pm 0.07$ & $2.45 \pm 0.82$ & $0.85 \pm 0.57$ & $0.46 \pm 0.05$ & $0.49 \pm 0.26$ & $1.98 \pm 1.08$ & $0.37 \pm 0.09$ & $0.39 \pm 0.16$ \\
\hline $20: 1 \mathrm{n} 9^{\mathrm{a}}$ & $0.64 \pm 0.58$ & $0.47 \pm 0.18$ & $0.94 \pm 0.21$ & $3.10 \pm 0.08$ & $7.46 \pm 1.60$ & $2.24 \pm 1.43$ & $1.21 \pm 0.13$ & $0.40 \pm 0.21$ \\
\hline 20:2 n6 & $0.44 \pm 0.06$ & $0.01 \pm 0.02$ & $1.22 \pm 0.18$ & $0.24 \pm 0.01$ & $0.27 \pm 0.10$ & $0.65 \pm 0.29$ & $1.07 \pm 0.17$ & $0.10 \pm 0.01$ \\
\hline 20:3 n6 & $0.76 \pm 0.08$ & $0.05 \pm 0.10$ & $0.55 \pm 0.24$ & $0.70 \pm 0.00$ & $0.18 \pm 0.07$ & $0.57 \pm 0.12$ & $0.22 \pm 0.04$ & $0.17 \pm 0.02$ \\
\hline $20: 4$ n6 (ARA) ${ }^{a}$ & $4.45 \pm 0.73$ & $1.03 \pm 0.31$ & $2.87 \pm 0.26$ & $1.14 \pm 0.03$ & $0.84 \pm 0.14$ & $2.07 \pm 0.56$ & $1.72 \pm 0.22$ & $1.17 \pm 0.03$ \\
\hline $21: 0^{\mathrm{a}}$ & $0.09 \pm 0.03$ & $0.04 \pm 0.01$ & $0.11 \pm 0.07$ & $0.07 \pm 0.01$ & $0.04 \pm 0.01$ & $0.04 \pm 0.02$ & $0.03 \pm 0.01$ & $0.02 \pm 0.01$ \\
\hline $20: 3 \mathrm{n} 3$ & $0.67 \pm 0.25$ & & $0.73 \pm 0.37$ & & & & $0.72 \pm 0.05$ & $0.09 \pm 0.02$ \\
\hline $20: 4$ n3 & $1.22 \pm 0.21$ & $0.25 \pm 0.12$ & $0.42 \pm 0.10$ & $1.17 \pm 0.07$ & $0.63 \pm 0.17$ & $0.54 \pm 0.27$ & $1.04 \pm 0.21$ & $0.71 \pm 0.14$ \\
\hline $20: 5 \mathrm{n} 3(\mathrm{EPA})^{\mathrm{a}}$ & $5.52 \pm 1.80$ & $4.67 \pm 1.67$ & $8.08 \pm 3.05$ & $13.46 \pm 0.84$ & $8.86 \pm 0.78$ & $13.76 \pm 5.14$ & $23.12 \pm 1.53$ & $19.48 \pm 1.01$ \\
\hline $22: 0^{\mathrm{a}}$ & $0.11 \pm 0.01$ & $0.12 \pm 0.03$ & $0.18 \pm 0.11$ & $0.09 \pm 0.01$ & $0.08 \pm 0.01$ & $0.15 \pm 0.07$ & $0.05 \pm 0.00$ & $0.05 \pm 0.01$ \\
\hline 22:1 n9 & $0.41 \pm 0.45$ & $0.22 \pm 0.10$ & $0.20 \pm 0.11$ & $1.84 \pm 0.07$ & $13.79 \pm 3.13$ & $0.28 \pm 0.14$ & $0.17 \pm 0.04$ & $0.09 \pm 0.05$ \\
\hline $22: 26^{a}$ & $0.00 \pm 0.00$ & $0.00 \pm 0.00$ & $0.14 \pm 0.09$ & $0.71 \pm 0.04$ & $0.26 \pm 0.14$ & $0.20 \pm 0.10$ & $0.31 \pm 0.07$ & $0.88 \pm 0.05$ \\
\hline 22:5 n3 (DPA) & $1.64 \pm 0.32$ & $0.00 \pm 0.00$ & $0.57 \pm 0.30$ & $1.81 \pm 0.08$ & $0.78 \pm 0.24$ & $1.12 \pm 0.41$ & $0.65 \pm 0.11$ & $0.51 \pm 0.05$ \\
\hline $22: 6$ n3 (DHA) ${ }^{\mathrm{a}}$ & $11.05 \pm 3.01$ & $0.00 \pm 0.00$ & $1.95 \pm 0.82$ & $13.29 \pm 0.71$ & $19.46 \pm 4.77$ & $8.95 \pm 2.84$ & $19.94 \pm 1.52$ & $13.16 \pm 1.08$ \\
\hline $24: 0^{\mathrm{a}}$ & $0.00 \pm 0.00$ & $0.00 \pm 0.00$ & $0.00 \pm 0.00$ & $0.25 \pm 0.04$ & $0.21 \pm 0.11$ & $0.21 \pm 0.30$ & $0.23 \pm 0.06$ & $0.05 \pm 0.05$ \\
\hline $24: 1 \mathrm{n} 9^{\mathrm{a}}$ & $0.00 \pm 0.00$ & $0.00 \pm 0.00$ & $0.00 \pm 0.00$ & $0.39 \pm 0.02$ & $0.43 \pm 0.15$ & $0.00 \pm 0.00$ & $0.16 \pm 0.04$ & $0.04 \pm 0.05$ \\
\hline$\sum \mathrm{sat}^{\mathrm{a}}$ & $45.98 \pm 4.98$ & $42.58 \pm 4.06$ & $29.92 \pm 5.80$ & $29.47 \pm 2.37$ & $28.05 \pm 6.01$ & $38.00 \pm 6.50$ & $27.25 \pm 3.03$ & $28.32 \pm 0.91$ \\
\hline$\sum 1$ unsat & $21.55 \pm 5.22$ & $35.86 \pm 1.66$ & $36.12 \pm 3.49$ & $28.58 \pm 0.37$ & $36.21 \pm 7.27$ & $29.70 \pm 3.31$ & $20.30 \pm 0.86$ & $24.43 \pm 1.73$ \\
\hline$\sum 2$ unsat $^{\mathrm{a}}$ & $4.23 \pm 0.62$ & $13.94 \pm 2.64$ & $12.31 \pm 1.29$ & $6.59 \pm 0.53$ & $2.61 \pm 0.62$ & $3.10 \pm 0.37$ & $3.20 \pm 0.25$ & $3.71 \pm 0.27$ \\
\hline$\sum 3$ unsat & $4.08 \pm 1.25$ & $1.57 \pm 0.20$ & $7.55 \pm 2.58$ & $2.51 \pm 0.10$ & $1.51 \pm 0.57$ & $2.65 \pm 0.99$ & $2.19 \pm 0.33$ & $3.84 \pm 0.23$ \\
\hline$\sum 4+$ unsat $^{\mathrm{a}}$ & $24.21 \pm 5.73$ & $6.05 \pm 1.99$ & $14.26 \pm 4.27$ & $32.89 \pm 1.66$ & $31.81 \pm 3.90$ & $26.61 \pm 8.72$ & $47.16 \pm 2.75$ & $39.74 \pm 1.18$ \\
\hline$\sum$ unsat $^{\mathrm{a}}$ & $54.07 \pm 5.01$ & $57.42 \pm 4.06$ & $70.23 \pm 5.64$ & $70.57 \pm 2.36$ & $72.14 \pm 5.92$ & $62.06 \pm 6.49$ & $72.85 \pm 3.05$ & $71.73 \pm 0.89$ \\
\hline sat:unsat ${ }^{a}$ & $0.86 \pm 0.19$ & $0.75 \pm 0.12$ & $0.43 \pm 0.12$ & $0.42 \pm 0.05$ & $0.40 \pm 0.11$ & $0.63 \pm 0.17$ & $0.38 \pm 0.06$ & $0.39 \pm 0.02$ \\
\hline$\sum \mathrm{n} 3^{\mathrm{a}}$ & $22.57 \pm 5.18$ & $5.78 \pm 1.69$ & $17.38 \pm 5.88$ & $32.82 \pm 1.71$ & $31.88 \pm 3.59$ & $25.98 \pm 7.95$ & $47.07 \pm 2.39$ & $40.94 \pm 1.26$ \\
\hline$\sum \mathrm{n} 6^{\mathrm{a}}$ & $9.06 \pm 0.54$ & $14.45 \pm 2.72$ & $15.32 \pm 1.83$ & $7.95 \pm 0.52$ & $3.49 \pm 0.56$ & $5.39 \pm 0.62$ & $4.99 \pm 0.31$ & $4.61 \pm 0.12$ \\
\hline$n 3: 6^{a}$ & $2.47 \pm 0.43$ & $0.39 \pm 0.07$ & $1.15 \pm 0.42$ & $4.13 \pm 0.18$ & $9.44 \pm 2.54$ & $4.77 \pm 1.17$ & $9.46 \pm 0.57$ & $8.89 \pm 0.16$ \\
\hline$\sum \mathrm{C} 16$ PUFA & $0.59 \pm 0.52$ & $0.98 \pm 0.31$ & $0.97 \pm 0.19$ & $0.97 \pm 0.06$ & $0.46 \pm 0.16$ & $0.74 \pm 0.22$ & $0.36 \pm 0.03$ & $1.65 \pm 0.26$ \\
\hline$\sum \mathrm{C} 18 \mathrm{PUFA}^{\mathrm{a}}$ & $6.18 \pm 1.67$ & $14.56 \pm 2.45$ & $16.59 \pm 2.73$ & $8.37 \pm 0.66$ & $4.02 \pm 1.26$ & $3.53 \pm 1.04$ & $3.39 \pm 0.50$ & $9.37 \pm 0.67$ \\
\hline$\sum \mathrm{C} 20 \mathrm{PUFA}^{\mathrm{a}}$ & $13.05 \pm 2.73$ & $6.03 \pm 2.04$ & $13.88 \pm 3.49$ & $16.84 \pm 0.82$ & $10.95 \pm 0.99$ & $17.82 \pm 5.68$ & $27.89 \pm 1.67$ & $21.73 \pm 0.87$ \\
\hline$\sum \mathrm{C} 22 \mathrm{PUFA}^{\mathrm{a}}$ & $12.69 \pm 3.30$ & $0.00 \pm 0.00$ & $2.67 \pm 1.11$ & $15.81 \pm 0.81$ & $20.50 \pm 4.54$ & $10.27 \pm 3.30$ & $20.91 \pm 1.38$ & $14.56 \pm 1.09$ \\
\hline$\sum$ PUFA $^{\mathrm{a}}$ & $32.51 \pm 5.35$ & $21.56 \pm 4.61$ & $34.11 \pm 5.66$ & $41.99 \pm 2.15$ & $35.93 \pm 3.05$ & $32.36 \pm 8.32$ & $52.55 \pm 2.53$ & $47.30 \pm 1.41$ \\
\hline $\mathrm{EPA} \mathrm{ARA}^{\mathrm{a}}$ & $1.23 \pm 0.29$ & $4.44 \pm 0.63$ & $2.79 \pm 0.87$ & $11.84 \pm 0.50$ & $10.87 \pm 2.93$ & $6.74 \pm 2.22$ & $13.53 \pm 1.02$ & $16.66 \pm 1.11$ \\
\hline DHA:EPA ${ }^{a}$ & $2.06 \pm 0.37$ & $0.00 \pm 0.00$ & $0.25 \pm 0.09$ & $0.99 \pm 0.04$ & $2.23 \pm 0.61$ & $0.66 \pm 0.06$ & $0.86 \pm 0.03$ & $0.68 \pm 0.08$ \\
\hline
\end{tabular}


Table 5 Fatty acids ( $\%$ of total FAC \pm SD) in muscle of juvenile eels reared on different diets; "sat" = saturated fatty acids; "unsat" = unsaturated fatty acids; "PUFA" = poly unsaturated fatty acids; $\mathrm{a}=t$-test identified a significant difference $(P<0.05)$ between freshwater (Rutilus rutilus, Chironomidae larvae, and Gammarus pulex) and marine treatments (Pellets, Clupea harengus, Mysis spec., and Euphausia superba)

\begin{tabular}{|c|c|c|c|c|c|c|c|c|}
\hline & $\begin{array}{l}\text { Eel Rutilus } \\
(N=12)\end{array}$ & $\begin{array}{l}\text { Eel Ch.larvae } \\
(N=12)\end{array}$ & $\begin{array}{l}\text { Eel Gammarus } \\
(N=12)\end{array}$ & $\begin{array}{l}\text { Eel Pellets } \\
(N=12)\end{array}$ & $\begin{array}{l}\text { Eel Clupea } \\
(N=12)\end{array}$ & $\begin{array}{l}\text { Eel Crangon } \\
(N=12)\end{array}$ & $\begin{array}{l}\text { Eel Mysis } \\
(N=12)\end{array}$ & $\begin{array}{l}\text { Eel Euphausic } \\
(N=12)\end{array}$ \\
\hline $16: 0^{\mathrm{a}}$ & $13.76 \pm 0.74$ & $12.28 \pm 0.75$ & $12.60 \pm 1.10$ & $15.69 \pm 1.51$ & $13.89 \pm 1.10$ & $15.77 \pm 0.53$ & $15.41 \pm 1.40$ & $14.30 \pm 0.89$ \\
\hline $16: 1 \mathrm{n} 7^{\mathrm{a}}$ & $5.11 \pm 0.26$ & $4.26 \pm 0.26$ & $4.65 \pm 0.63$ & $5.69 \pm 0.61$ & $5.04 \pm 0.33$ & $4.90 \pm 0.62$ & $5.23 \pm 0.29$ & $5.10 \pm 0.46$ \\
\hline $16: 2 \mathrm{n} 4$ & $0.22 \pm 0.00$ & $0.20 \pm 0.01$ & $0.24 \pm 0.02$ & $0.29 \pm 0.03$ & $0.22 \pm 0.05$ & $0.23 \pm 0.02$ & $0.24 \pm 0.02$ & $0.24 \pm 0.04$ \\
\hline $16: 3 \mathrm{n} 4$ & $0.24 \pm 0.03$ & $0.20 \pm 0.01$ & $0.24 \pm 0.03$ & $0.26 \pm 0.03$ & $0.24 \pm 0.02$ & $0.22 \pm 0.04$ & $0.23 \pm 0.02$ & $0.24 \pm 0.01$ \\
\hline $17: 0^{\mathrm{a}}$ & $0.30 \pm 0.02$ & $0.31 \pm 0.02$ & $0.35 \pm 0.05$ & $0.36 \pm 0.06$ & $0.31 \pm 0.03$ & $0.43 \pm 0.05$ & $0.36 \pm 0.02$ & $0.35 \pm 0.04$ \\
\hline $17: 1 \mathrm{n} 7^{\mathrm{a}}$ & $0.26 \pm 0.01$ & $0.23 \pm 0.01$ & $0.24 \pm 0.01$ & $0.27 \pm 0.02$ & $0.28 \pm 0.02$ & $0.30 \pm 0.02$ & $0.25 \pm 0.01$ & $0.26 \pm 0.01$ \\
\hline 18:0 & $3.50 \pm 0.33$ & $3.31 \pm 0.24$ & $4.02 \pm 0.98$ & $3.28 \pm 0.12$ & $2.83 \pm 0.25$ & $4.28 \pm 1.67$ & $3.46 \pm 0.49$ & $3.19 \pm 0.43$ \\
\hline 18:1 n9 & $28.87 \pm 1.86$ & $26.13 \pm 1.53$ & $26.70 \pm 1.77$ & $26.54 \pm 1.98$ & $25.26 \pm 1.51$ & $29.28 \pm 1.54$ & $26.14 \pm 0.49$ & $26.67 \pm 1.01$ \\
\hline 18:1 n7 & $0.46 \pm 0.02$ & $0.45 \pm 0.02$ & $0.45 \pm 0.02$ & $0.46 \pm 0.03$ & $0.45 \pm 0.02$ & $0.44 \pm 0.06$ & $0.48 \pm 0.01$ & $0.47 \pm 0.02$ \\
\hline $18: 2 \mathrm{n}^{\mathrm{a}}$ & $4.42 \pm 0.35$ & $4.24 \pm 0.16$ & $4.98 \pm 0.33$ & $4.40 \pm 0.10$ & $3.93 \pm 0.11$ & $4.32 \pm 0.50$ & $4.20 \pm 0.30$ & $4.40 \pm 0.13$ \\
\hline 18:3 n6 & $0.12 \pm 0.01$ & $0.17 \pm 0.12$ & $0.16 \pm 0.06$ & $0.11 \pm 0.03$ & $0.10 \pm 0.02$ & $0.11 \pm 0.02$ & $0.12 \pm 0.02$ & $0.13 \pm 0.01$ \\
\hline $18: 3 \mathrm{n} 4$ & $0.18 \pm 0.01$ & $0.17 \pm 0.00$ & $0.21 \pm 0.03$ & $0.26 \pm 0.05$ & $0.19 \pm 0.01$ & $0.18 \pm 0.01$ & $0.18 \pm 0.01$ & $0.18 \pm 0.01$ \\
\hline $18: 3 \mathrm{n} 3$ & $0.72 \pm 0.05$ & $0.51 \pm 0.09$ & $0.73 \pm 0.06$ & $0.72 \pm 0.10$ & $0.62 \pm 0.06$ & $0.63 \pm 0.10$ & $0.66 \pm 0.09$ & $0.67 \pm 0.18$ \\
\hline 18:4 n3 & $0.51 \pm 0.06$ & $0.50 \pm 0.13$ & $0.55 \pm 0.02$ & $0.69 \pm 0.15$ & $0.57 \pm 0.03$ & $0.48 \pm 0.09$ & $0.59 \pm 0.11$ & $0.63 \pm 0.05$ \\
\hline 20:0 & $0.25 \pm 0.02$ & $0.93 \pm 1.16$ & $0.36 \pm 0.06$ & $0.24 \pm 0.06$ & $0.24 \pm 0.03$ & $0.41 \pm 0.10$ & $0.27 \pm 0.06$ & $0.29 \pm 0.13$ \\
\hline $20: 1 \mathrm{n} 9^{\mathrm{a}}$ & $17.91 \pm 1.01$ & $20.02 \pm 0.93$ & $18.52 \pm 1.80$ & $13.61 \pm 5.48$ & $17.62 \pm 0.90$ & $13.54 \pm 4.57$ & $17.17 \pm 1.70$ & $17.47 \pm 1.03$ \\
\hline $20: 2 n 6^{a}$ & $0.46 \pm 0.01$ & $0.45 \pm 0.03$ & $0.47 \pm 0.01$ & $0.44 \pm 0.02$ & $0.42 \pm 0.01$ & $0.42 \pm 0.06$ & $0.43 \pm 0.01$ & $0.40 \pm 0.02$ \\
\hline $20: 3 n 6^{a}$ & $0.97 \pm 0.10$ & $1.03 \pm 0.06$ & $1.15 \pm 0.22$ & $0.89 \pm 0.04$ & $0.69 \pm 0.12$ & $0.97 \pm 0.06$ & $0.86 \pm 0.09$ & $0.89 \pm 0.08$ \\
\hline 20:4 n6 (ARA) & $0.82 \pm 0.04$ & $0.76 \pm 0.07$ & $0.86 \pm 0.18$ & $0.69 \pm 0.06$ & $0.74 \pm 0.11$ & $0.94 \pm 0.13$ & $0.72 \pm 0.08$ & $0.71 \pm 0.08$ \\
\hline 21:0 & $0.02 \pm 0.01$ & $0.02 \pm 0.01$ & $0.02 \pm 0.00$ & $0.03 \pm 0.00$ & $0.03 \pm 0.01$ & $0.03 \pm 0.00$ & $0.03 \pm 0.01$ & $0.02 \pm 0.01$ \\
\hline $20: 3 \mathrm{n} 3$ & $0.19 \pm 0.02$ & $0.13 \pm 0.03$ & $0.15 \pm 0.02$ & $0.16 \pm 0.02$ & $0.19 \pm 0.02$ & $0.13 \pm 0.03$ & $0.16 \pm 0.02$ & $0.14 \pm 0.01$ \\
\hline $20: 4$ n3 & $1.52 \pm 0.09$ & $1.47 \pm 0.22$ & $1.46 \pm 0.08$ & $1.68 \pm 0.19$ & $1.60 \pm 0.07$ & $1.22 \pm 0.08$ & $1.49 \pm 0.10$ & $1.60 \pm 0.10$ \\
\hline $20: 5 \mathrm{n} 3(\mathrm{EPA})^{\mathrm{a}}$ & $3.04 \pm 0.52$ & $3.17 \pm 0.77$ & $3.18 \pm 0.11$ & $4.34 \pm 0.89$ & $4.13 \pm 0.28$ & $3.69 \pm 0.59$ & $4.04 \pm 0.51$ & $3.87 \pm 0.36$ \\
\hline 22:0 & $0.03 \pm 0.01$ & $0.04 \pm 0.00$ & $0.06 \pm 0.02$ & $0.03 \pm 0.01$ & $0.03 \pm 0.01$ & $0.05 \pm 0.00$ & $0.03 \pm 0.01$ & $0.03 \pm 0.01$ \\
\hline $22: 1 \mathrm{n} 9^{\mathrm{a}}$ & $6.25 \pm 0.76$ & $7.56 \pm 0.52$ & $7.31 \pm 1.40$ & $6.06 \pm 0.79$ & $7.00 \pm 1.12$ & $5.63 \pm 1.12$ & $5.67 \pm 0.42$ & $5.89 \pm 0.48$ \\
\hline $22: 2 n 6^{a}$ & $0.23 \pm 0.03$ & $0.27 \pm 0.05$ & $0.24 \pm 0.05$ & $0.36 \pm 0.05$ & $0.31 \pm 0.03$ & $0.22 \pm 0.05$ & $0.28 \pm 0.03$ & $0.30 \pm 0.03$ \\
\hline $22: 5$ n3 (DPA) & $1.98 \pm 0.10$ & $2.31 \pm 0.33$ & $2.17 \pm 0.42$ & $2.42 \pm 0.34$ & $2.48 \pm 0.41$ & $2.30 \pm 0.63$ & $2.25 \pm 0.07$ & $2.33 \pm 0.17$ \\
\hline $22: 6 \mathrm{n} 3(\mathrm{DHA})^{\mathrm{a}}$ & $7.25 \pm 0.89$ & $8.34 \pm 1.85$ & $7.35 \pm 0.88$ & $9.64 \pm 1.53$ & $10.13 \pm 0.70$ & $8.40 \pm 1.95$ & $8.73 \pm 0.83$ & $8.82 \pm 0.49$ \\
\hline $24: 0$ & $0.07 \pm 0.02$ & $0.10 \pm 0.01$ & $0.09 \pm 0.03$ & $0.10 \pm 0.01$ & $0.10 \pm 0.02$ & $0.13 \pm 0.02$ & $0.09 \pm 0.02$ & $0.10 \pm 0.02$ \\
\hline $24: 1 \operatorname{n} 9^{a}$ & $0.33 \pm 0.03$ & $0.43 \pm 0.04$ & $0.50 \pm 0.11$ & $0.29 \pm 0.09$ & $0.36 \pm 0.12$ & $0.35 \pm 0.11$ & $0.24 \pm 0.04$ & $0.24 \pm 0.04$ \\
\hline$\sum \mathrm{sat}^{\mathrm{a}}$ & $17.98 \pm 0.59$ & $17.02 \pm 1.07$ & $17.54 \pm 1.71$ & $19.76 \pm 1.42$ & $17.49 \pm 1.26$ & $21.17 \pm 2.28$ & $19.69 \pm 1.98$ & $18.33 \pm 1.52$ \\
\hline$\sum 1$ unsat $^{\mathrm{a}}$ & $59.20 \pm 1.41$ & $59.08 \pm 2.61$ & $58.37 \pm 1.94$ & $52.92 \pm 3.95$ & $56.01 \pm 0.86$ & $54.43 \pm 1.68$ & $55.17 \pm 1.83$ & $56.14 \pm 2.27$ \\
\hline$\sum 2$ unsat & $5.33 \pm 0.31$ & $5.15 \pm 0.22$ & $5.94 \pm 0.29$ & $5.50 \pm 0.13$ & $4.89 \pm 0.16$ & $5.19 \pm 0.59$ & $5.15 \pm 0.29$ & $5.35 \pm 0.12$ \\
\hline$\sum 3$ unsat $^{\mathrm{a}}$ & $2.42 \pm 0.09$ & $2.22 \pm 0.16$ & $2.63 \pm 0.21$ & $2.41 \pm 0.13$ & $2.03 \pm 0.15$ & $2.24 \pm 0.07$ & $2.21 \pm 0.08$ & $2.25 \pm 0.13$ \\
\hline$\sum 4+$ unsat $^{\mathrm{a}}$ & $15.13 \pm 1.55$ & $16.55 \pm 3.16$ & $15.57 \pm 1.24$ & $19.45 \pm 2.98$ & $19.65 \pm 1.30$ & $17.03 \pm 3.35$ & $17.82 \pm 1.65$ & $17.97 \pm 0.76$ \\
\hline$\sum$ unsat $^{\mathrm{a}}$ & $82.07 \pm 0.57$ & $83.01 \pm 1.07$ & $82.51 \pm 1.71$ & $80.28 \pm 1.43$ & $82.58 \pm 1.29$ & $78.90 \pm 2.25$ & $80.35 \pm 1.96$ & $81.71 \pm 1.50$ \\
\hline sat:unsat $^{\mathrm{a}}$ & $0.22 \pm 0.01$ & $0.21 \pm 0.02$ & $0.21 \pm 0.03$ & $0.25 \pm 0.02$ & $0.21 \pm 0.02$ & $0.27 \pm 0.04$ & $0.25 \pm 0.03$ & $0.23 \pm 0.02$ \\
\hline$\sum \mathrm{n} 3^{\mathrm{a}}$ & $15.22 \pm 1.59$ & $16.44 \pm 3.30$ & $15.58 \pm 1.21$ & $19.64 \pm 3.05$ & $19.71 \pm 1.30$ & $16.86 \pm 3.26$ & $17.92 \pm 1.66$ & $18.06 \pm 0.96$ \\
\hline$\sum \mathrm{n} 6^{\mathrm{a}}$ & $7.02 \pm 0.45$ & $6.92 \pm 0.14$ & $7.87 \pm 0.59$ & $6.90 \pm 0.17$ & $6.21 \pm 0.15$ & $6.98 \pm 0.67$ & $6.62 \pm 0.44$ & $6.84 \pm 0.26$ \\
\hline $\mathrm{n} 3: \mathrm{n} 6^{\mathrm{a}}$ & $2.19 \pm 0.35$ & $2.37 \pm 0.44$ & $2.01 \pm 0.21$ & $2.85 \pm 0.41$ & $3.20 \pm 0.25$ & $2.41 \pm 0.48$ & $2.71 \pm 0.31$ & $2.65 \pm 0.23$ \\
\hline$\sum$ C16 PUFA & $0.46 \pm 0.03$ & $0.40 \pm 0.02$ & $0.48 \pm 0.04$ & $0.56 \pm 0.06$ & $0.46 \pm 0.04$ & $0.45 \pm 0.05$ & $0.47 \pm 0.03$ & $0.48 \pm 0.04$ \\
\hline$\sum \mathrm{C} 18$ PUFA & $5.95 \pm 0.25$ & $5.59 \pm 0.35$ & $6.63 \pm 0.23$ & $6.19 \pm 0.33$ & $5.41 \pm 0.10$ & $5.72 \pm 0.44$ & $5.75 \pm 0.27$ & $6.01 \pm 0.21$ \\
\hline$\sum \mathrm{C} 20 \mathrm{PUFA}^{\mathrm{a}}$ & $6.99 \pm 0.53$ & $7.01 \pm 0.91$ & $7.27 \pm 0.37$ & $8.20 \pm 1.15$ & $7.78 \pm 0.43$ & $7.37 \pm 0.84$ & $7.70 \pm 0.63$ & $7.62 \pm 0.27$ \\
\hline$\sum$ C22 PUFA ${ }^{\mathrm{a}}$ & $9.47 \pm 0.96$ & $10.92 \pm 2.16$ & $9.76 \pm 1.25$ & $12.42 \pm 1.91$ & $12.93 \pm 1.13$ & $10.93 \pm 2.62$ & $11.26 \pm 0.93$ & $11.46 \pm 0.41$ \\
\hline$\sum$ PUFA $^{\mathrm{a}}$ & $22.87 \pm 1.26$ & $23.93 \pm 3.39$ & $24.14 \pm 1.36$ & $27.36 \pm 3.17$ & $26.57 \pm 1.33$ & $24.46 \pm 3.58$ & $25.18 \pm 1.72$ & $25.57 \pm 0.81$ \\
\hline $\mathrm{EPA} \mathrm{ARA}^{\mathrm{a}}$ & $3.84 \pm 0.85$ & $4.32 \pm 1.08$ & $3.95 \pm 0.42$ & $6.32 \pm 1.12$ & $5.72 \pm 0.77$ & $4.14 \pm 0.32$ & $5.88 \pm 0.99$ & $5.58 \pm 0.93$ \\
\hline DHA:EPA ${ }^{\mathrm{a}}$ & $2.44 \pm 0.17$ & $2.74 \pm 0.05$ & $2.36 \pm 0.32$ & $2.26 \pm 0.25$ & $2.48 \pm 0.19$ & $2.25 \pm 0.21$ & $2.17 \pm 0.09$ & $2.32 \pm 0.07$ \\
\hline
\end{tabular}




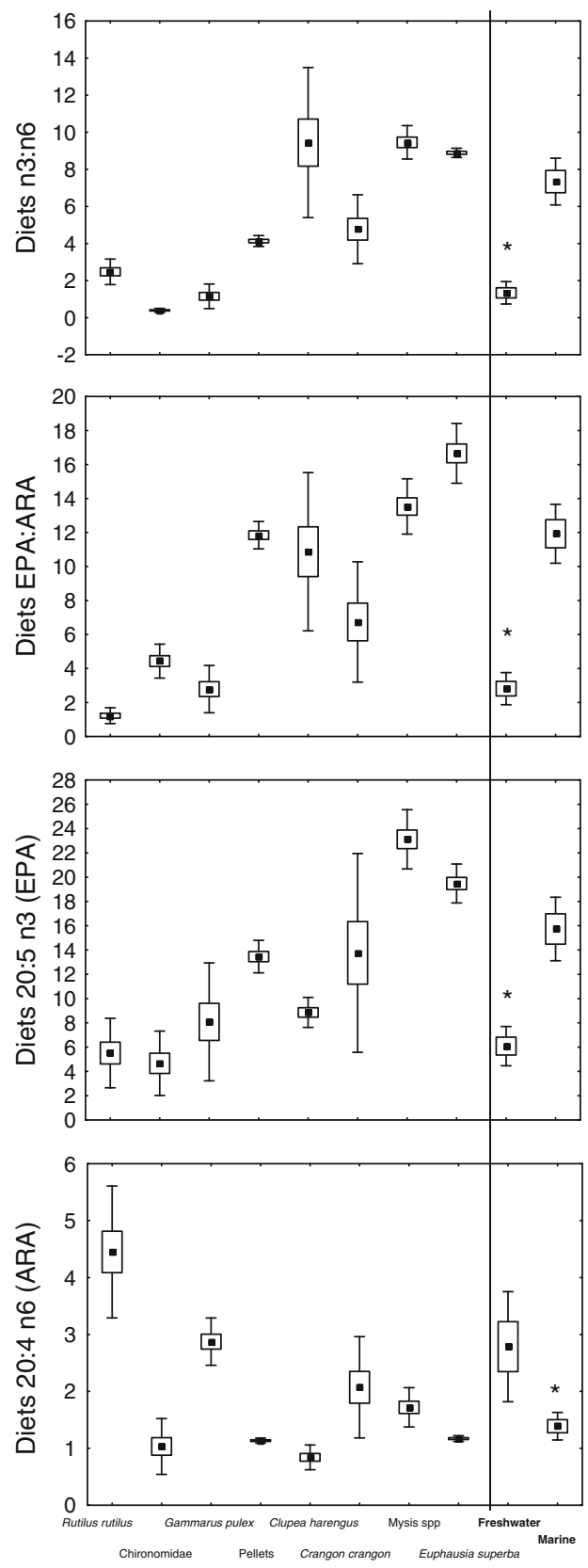

Fig. 1 Fatty acids (\% of total $F A C)$ and fatty acid ratios in diet samples and muscle tissue of juvenile eels. Dot $=$ mean value, box $= \pm$ standard deviation, whiskers $= \pm 0.95$ confidence interval;

\section{Discussion}

Growth performance in the present study was in the same magnitude as previously reported by de la Higuera et al. (1999). They documented SGRs (\%weight day ${ }^{-1}$ ) in European eel fed for 12 weeks with different artificial diets to be ranging between 0.04 and $0.39 \%$ day $^{-1}$. Even though most of the recorded SGRs in this experiment were in the same range, two of the present treatments (G. pulex and E. superba) resulted in negative SGRs. Possible reasons for

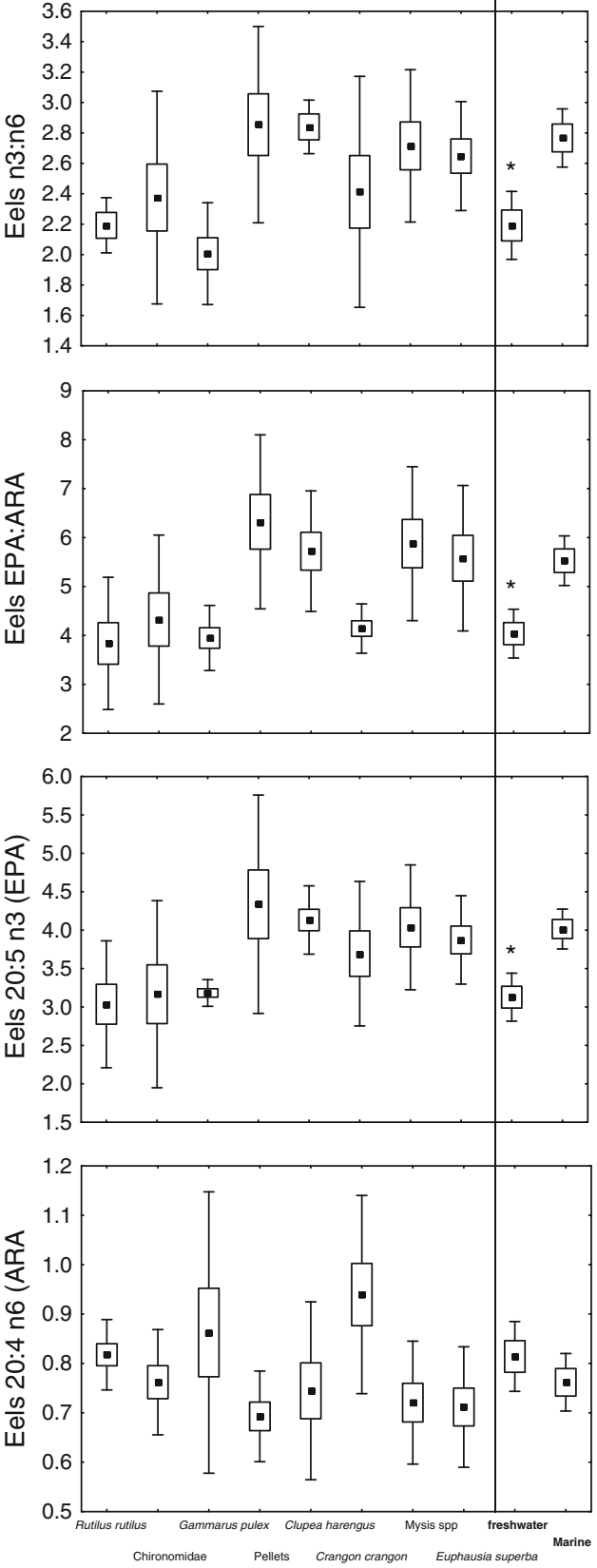

"Freshwater" and "Marine" are mean values of different diet-habitats; Significant differences between diet-habitats are indicated by*

negative weight development include insufficient food quality, adaptations to the experimental environment, and interactions between individuals. Knights (1983) and Wickins (1985) described hierarchical behavior in European eel to be enhanced at low rearing densities. However, weight development of eels in the tanks of the two treatments with negative mean SGR (G. pulex and E. superba) does not support hierarchical interactions being a possible reason for the poor growth. Individual growth performance does not indicate a single eel dominating the rest in the G. pulex and 
Table 6 Principal component analysis of FAC in diets and eels; trait loadings on the first axis; shown are correlation coefficients with the first axis

\begin{tabular}{|c|c|c|}
\hline & Diets & Eels \\
\hline Variance explained* & $39 \%$ & $30 \%$ \\
\hline $16: 0$ & 0.17 & 0.04 \\
\hline $16: 1 \mathrm{n} 7$ & -0.32 & 0.33 \\
\hline $16: 2 \mathrm{n} 4$ & -0.67 & 0.28 \\
\hline $16: 3 \mathrm{n} 4$ & 0.03 & 0.10 \\
\hline $17: 0$ & -0.33 & -0.02 \\
\hline 17:1 n7 & -0.61 & 0.23 \\
\hline 18:0 & -0.25 & -0.36 \\
\hline 18:1 n9 & -0.75 & -0.49 \\
\hline 18:1 n7 & -0.27 & -0.10 \\
\hline 18:2 n6 & -0.95 & -0.06 \\
\hline $18: 3$ n6 & -0.50 & 0.10 \\
\hline $18: 3$ n4 & -0.76 & 0.20 \\
\hline 18:3 n3 (alpha-linoleic) & -0.31 & 0.25 \\
\hline $18: 4$ n3 & 0.34 & 0.68 \\
\hline 20:0 & -0.39 & -0.16 \\
\hline 20:1 n9 & 0.25 & -0.45 \\
\hline 20:2 n6 & 0.07 & -0.03 \\
\hline $20: 3$ n6 & 0.08 & -0.30 \\
\hline 20:4 n6 (ARA) & -0.04 & 0.06 \\
\hline $21: 0$ & -0.33 & 0.15 \\
\hline $20: 3$ n3 & 0.09 & 0.55 \\
\hline $20: 4 \mathrm{n} 3$ & 0.40 & 0.65 \\
\hline 20:5 n3 (EPA) & 0.47 & 0.91 \\
\hline $22: 0$ & -0.39 & -0.22 \\
\hline 22:1 n9 & 0.26 & -0.20 \\
\hline 22:2 n6 & 0.35 & 0.72 \\
\hline 22:5 n3 (DPA) & 0.42 & 0.77 \\
\hline 22:3 n3 (DHA) & 0.83 & 0.96 \\
\hline $24: 0$ & 0.44 & 0.22 \\
\hline 24:1 n9 & 0.28 & -0.07 \\
\hline SATT & -0.10 & -0.15 \\
\hline lun & -0.56 & -0.75 \\
\hline 2 un & -0.97 & 0.10 \\
\hline 3 un & -0.30 & 0.08 \\
\hline $4+$ un & 0.74 & 0.97 \\
\hline Sum uns & 0.10 & 0.15 \\
\hline SAT/UNSAT & -0.09 & -0.16 \\
\hline n3 & 0.70 & 0.97 \\
\hline n6 & -0.91 & 0.00 \\
\hline $\mathrm{n} 3 / \mathrm{n} 6$ & 0.84 & 0.88 \\
\hline SUM C16 PUFA & -0.35 & 0.26 \\
\hline SUM C18 PUFA & -0.84 & 0.24 \\
\hline SUM C20 PUFA & 0.47 & 0.90 \\
\hline SUM C22 PUFA & 0.82 & 0.96 \\
\hline SUM PUFA & 0.47 & 0.94 \\
\hline EPA/ARA & 0.43 & 0.69 \\
\hline DHA/EPA & 0.73 & -0.13 \\
\hline
\end{tabular}

* Variance explained by the first axis
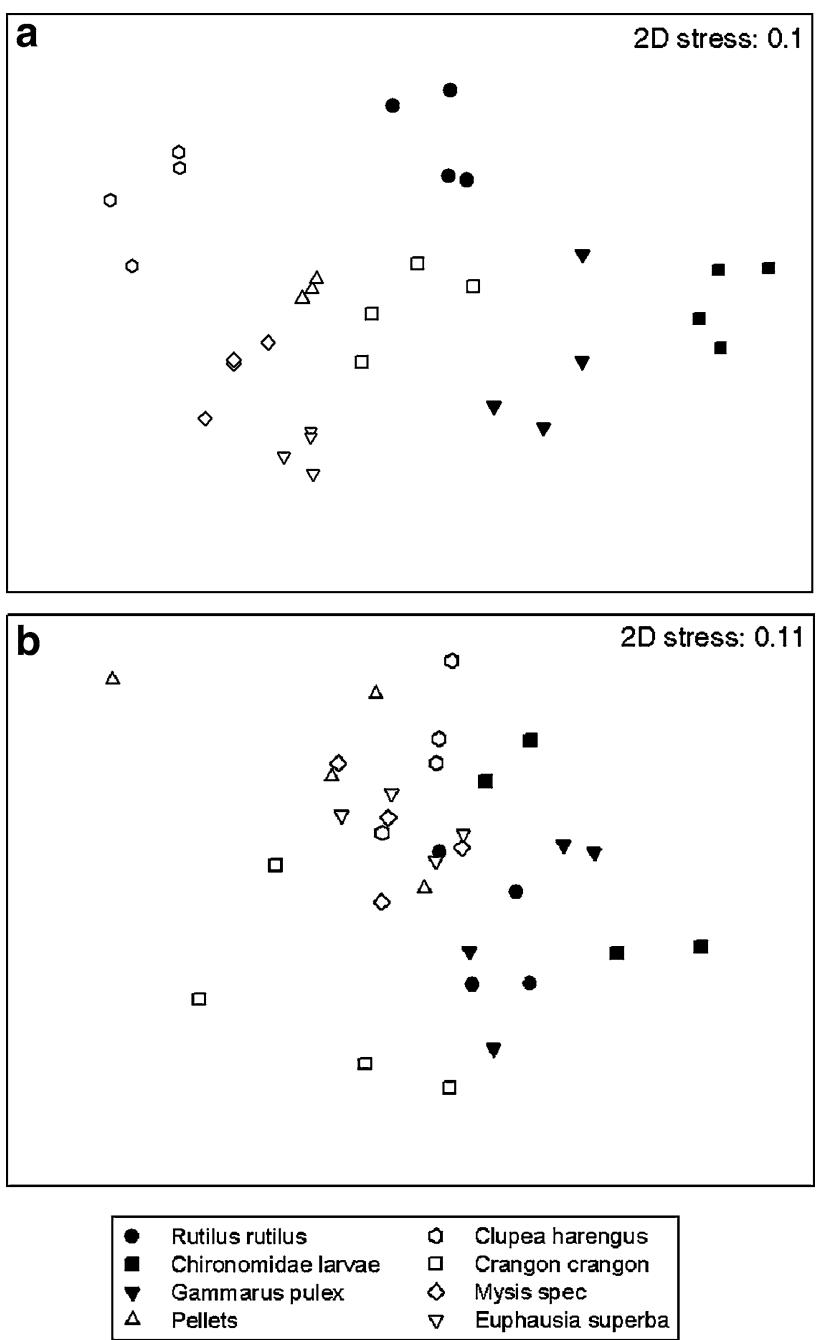

Fig. 2 MDS plot of FAC of (a) diets and (b) eels; freshwater diets $=$ black $;$ marine diets $=$ white

E. superba tanks. Thus, negative SGR was most likely due to insufficient food quality. Nevertheless, as all eels seemed to be feeding, an at least partial replacement of fatty acids in the muscle tissue was assumed. Therefore, none of the groups were excluded from analysis.

Several studies have documented the influence of dietary lipid composition on the fatty acid composition in A. spp. (Vanvliet and Katan 1990; Agradi et al. 1995; Garcia-Gallego et al. 1999; Bae et al. 2010). However, these studies used artificially enriched diets, and it was often aimed at improving flesh quality for human consumption. Results were very variable, and lipid composition in muscle tissue of juvenile eels often seems to be rather conservative. This holds true for the present study with lipid composition in muscle tissue seemed to be rather insensitive to dietary FAC. Abrami et al. (1992) showed that wild eels have (I) significantly lower total lipid content and (II) significantly lower PUFA content than farmed eels. On strength of this difference 
between wild and farmed eels, Garcia-Gallego et al. (1999) are giving one possible reason for the present results. They suggested that the high-energy diet fed in fish farms prior to the experiment might have led to an extremely high muscle fat content in juvenile eels and thus inhibited the complete turnover of storage lipids. This point seems to apply also for our study. Considering the extensive stocking strategy in Europe where the large part of stocked materials being juvenile farmed eels, it seemed reasonable to use farmed eels instead of wild-caught eels in the present experiment. Furthermore, changes in fatty acid composition in fishes due to a change in diet are most likely a result of dilution. Stabilization of FAC after a diet-shift was reported to exceed our feeding period of 56 days in several carnivorous fish species (Regost et al. 2003; Robin et al. 2003; Jobling 2004). However, Elsdon (2010) was able to detect a significant influence of diet on FAC in black bream (Acanthopagrus butcheri) from a hatchery broodstock after a feeding period of only 42 days. It has to be noted that he was using juvenile fish with an initial size of $25 \mathrm{~mm}$ that most likely have much higher turnover of lipids. However the present analysis focused on PUFAs that are not usually used as energy reserves (Brett and Muller-Navarra 1997). Therefore, a complete turnover of storage lipids should not be essential for detecting the dietary influence.

In the present experiment, it was possible to recover some of the significant differences in FAC of the diets from freshwater and marine habitats in the muscle lipid composition of the respective eels. Especially the 20:5n-3 to $20: 4 n-6$ (EPA:ARA) ratio showed a clear pattern of higher ratios in eels reared on marine diets. It has previously been shown that the EPA:ARA ratio can be used as a bioindicator to differentiate between freshwater and marine animals (Innis et al. 1995; Steffens 1997; Diraman and Dibeklioglu 2009). The difference could be traced back to a substantially higher EPA levels in eels fed with marine diets. Higher EPA levels in marine organisms have previously been documented in several studies (Steffens 1997; Makhutova et al. 2003). EPA has been described as one of the most abundant PUFAs in marine Bacillariophyceae (Zhukova and Aizdaicher 1995), and Vanderploeg et al. (1996) documented an almost tenfold amount of EPA in marine green algae than in freshwater green algae.

It is noteworthy that both in the "Crangon crangon" diet and in eels fed with Crangon crangon, the EPA:ARA ratio was somehow an intermediate between freshwater diets/ eels and fully marine diets/eels, which might reflect the brackish habitat where $C$. crangon were captured.

One of the most commonly used bioindicators for the distinction between marine and freshwater organisms is the n3:n6 ratio, with marine organisms having substantially higher ratios than freshwater organisms (Steffens 1997). Analysis of dietary FAC supports those findings of higher $\mathrm{n} 3: \mathrm{n} 6$ ratios in marine food organism than in freshwater food organism. This pattern was again found in eels fed with different diets. Again, Crangon crangon and the eels fed with it showed an intermediate n3:n6 ratio between freshwater and fully marine treatments.

Regardless of dietary n3:n6 ratio, all eels maintained a ratio $<4$. Such a low ratio is commonly considered to be typical for freshwater fish (Innis et al. 1995). Furthermore, it is noteworthy that both $\Sigma \mathrm{n} 3$ and $\Sigma \mathrm{n} 6$ in eels of all treatments differed from the values in respective diets. Especially eels reared on Mysis spec., E. superba, and $C$. harengus must have taken up high amounts of $\mathrm{n} 3$ fatty acids (mainly EPA and DHA) with diets. However, substantially lower levels were detected in the respective eels. Thus, eels in the present study seemed to not unconditionally incorporate the complete amount of dietary $n 3$ and $n 6$ fatty acids into their muscle tissue, resulting in lower proportions of these fatty acid families in eels than in respective diets. Distinct differences between diet and consumer fatty acid composition have previously been reported by Christiansen et al. (1991) and Malzahn et al. (2010).

It has earlier been described that the essential fatty acid (EFA) requirements of freshwater fish can usually be met by dietary $18: 3 n-3$ and its conversion into 20:5n-3 (EPA) and 22:6n-3 (DHA) (Ackman 1967; Dave et al. 1976; Sargent et al. 1995). Christiansen et al. (1991) described that in rats, the conversion of $18: 3 \mathrm{n}-3$ to EPA and DHA is positively correlated to dietary $18: 3 n-3$ content but negatively to that of dietary EPA and DHA content. They suggest that the conversion of $18: 3 n-3$ is an optional pathway to ensure sufficient EPA and DHA supply. Applying this suggestion on the present study, the absence of DHA in Chironomidae larvae might have induced a high conversion of dietary 18:3n-3 into DHA in eels. Kanazawa et al. (1979) showed that Anguilla japonica is able to synthesize EPA and DHA from 18:3n-3. In addition, their results indicated a preferential synthesis of 18:3n-3 to DHA rather than to EPA. Our results support these findings with significantly higher DHA amounts in eels fed with Chironomidae larvae than that found in the diet. Therefore, it should be concluded that the European eel is capable of balancing low supply of certain polyunsaturated fatty acids (PUFA) by converting 18:3n-3 preferably into DHA that has just recently been described to play a more important role in fish growth than EPA (Boersma et al. 2009). Consequently, 18:3n-3 should be considered as an EFA for the European eel and needs to be taken up with the diet. This has previously been suggested for Anguilla japonica by Takeuchi et al. (1980).

It was possible to further illustrate differences in FAC between eels fed with marine and freshwater diets using multivariate approaches. In the MDS plot, two distinct groupings (freshwater and marine) are discernible. These results support the distinction of eels feeding in marine and 
freshwater habitats on the strength of their specific fatty acid composition.

Acknowledgments We thank Michael Gruber and Ralf Traulsen from Kiel Aquarium for their cooperation and advice. This study was funded by the German Federal Ministry of Consumer Protection, Food and Agriculture (BMELV) through the project "Habitat selection of the European eel” (04HS065).

\section{References}

Abrami G, Natiello F, Bronzi P, McKenzie D, Bolis L, Agradi E (1992) A comparison of highly unsaturated fatty acid levels in wild and farmed eels (Anguilla Anguilla). Comp Biochem Physiol B 101(1-2):79-81

Ackman RG (1967) Characteristics of the fatty acid composition and biochemistry of some fresh-water fish oils and lipids in comparison with marine oils and lipids. Comp Biochem Physiol 22(3):907-922

Agradi E, Bonomi L, Rigamonti E, Liguori M, Bronzi P (1995) The effect of dietary lipids on tissue-lipids and ammonia excretion in European eels (Anguilla anguilla). Comp Biochem Physiol A 111(3):445-451

Arim M, Naya DE (2003) Pinniped diets inferred from scats: analysis of biases in prey occurrence. Can J Zool 81(1):67-73

Bae JY, Kim DJ, Yoo KY, Kim SG, Lee JY, Bai SC (2010) Effects of dietary arachidonic acid (20:4n-6) levels on growth performance and fatty acid composition of juvenile eel, anguilla japonica. Asian-Aust J Anim Sci 23(4):508-514

Boersma M, Becker C, Malzahn AM, Vernooij S (2009) Food chain effects of nutrient limitation in primary producers. Mar Freshwater Res 60(10):983-989

Boggio SM, Hardy RW, Babbitt JK, Brannon EL (1985) The influence of dietary lipid source and alpha-tocopheryl acetate level on production quality of rainbow trout (Salmo gairdneri). Aquaculture 51(1):13-24

Bonhommeau S, Chassot E, Planque B, Rivot E, Knap AH, Le Pape O (2008) Impact of climate on eel populations of the Northern Hemisphere. Mar Ecol Prog Ser 373:71-80

Brett MT, Muller-Navarra DC (1997) The role of highly unsaturated fatty acids in aquatic food web processes. Freshwat Biol 38(3):483-499

Christiansen EN, Lund JS, Rortveit T, Rustan AC (1991) Effect of dietary n-3 and n-6 fatty acids on fatty acid desaturation in rat liver. Biochim Biophys Acta 1082(1):57-62

Cortes E (1997) A critical review of methods of studying fish feeding based on analysis of stomach contents: application to elasmobranch fishes. Can J Fish Aquat Sci 54(3):726-738

D'Abramo LR (1979) Dietary fatty acid and temperature effects on the productivity of the Cladoceran, Moina Macrocopa. Biol Bull 157:234-248

Dave G, Johansson-Sjöbeck ML, Larsson A, Lewander K, Lidman U (1976) Metabolic and hematological effects of starvation in European eel, Anguilla anguilla - L. 3. Fatty-acid composition. Comp Biochem Physiol B 53(4):509-515

Daverat F, Limburg KE, Thibault I, Shiao JC, Dodson JJ, Caron FO, Tzeng WN, Iizuka Y, Wickstrom H (2006) Phenotypic plasticity of habitat use by three temperate eel species, Anguilla anguilla, A-japonica and A-rostrata. Mar Ecol Prog Ser 308:231-241

de la Higuera M, Akharbach H, Hidalgo MC, Peragon J, Lupianez JA, Garcia-Gallego M (1999) Liver and white muscle protein turnover rates in the European eel (Anguilla anguilla): effects of dietary protein quality. Aquaculture 179(1-4):203-216
Dekker W (2003) On the distribution of the European eel (Anguilla anguilla) and its fisheries. Can J Fish Aquat Sci 60(7):787-799

Diraman H, Dibeklioglu H (2009) Chemometric characterization and classification of selected freshwater and marine fishes from Turkey based on their fatty acid profiles. J Am Oil Chem Soc 86(3):235-246

Elsdon TS (2010) Unraveling diet and feeding histories of fish using fatty acids as natural tracers. J Exp Mar Biol Ecol 386(1-2): $61-68$

Garcia-Gallego M, Akharbach H, de la Higuera M (1999) Effects of dietary lipid composition on growth, food utilization and body composition of European eel (Anguilla anguilla). Anim Sci 69(1):157-165

Harrod C, Grey J, McCarthy TK, Morrissey M (2005) Stable isotope analyses provide new insights into ecological plasticity in a mixohaline population of European eel. Oecologia 144(4):673-683

Henderson RJ, Tocher DR (1987) The lipid composition and biochemistry of fresh water fish. Prog Lipid Res 26(4):281-347

ICES (2002) Report of the ICES/EIFAC Working Group on Eels (WGEEL). International Council for the Exploration of the Sea

ICES (2006) Report of the ICES/EIFAC Working Group on Eels (WGEEL). International Council for the Exploration of the Sea

ICES (2008) Report of the ICES/EIFAC Working Group on Eels (WGEEL). International Council for the Exploration of the Sea

Innis SM, Rioux FM, Auestad N, Ackman RG (1995) Marine and freshwater fish oil varying in arachidonic, eicosapentaenoic and docosahexaenoic acids differ in their effects on organ lipids and fatty acids in growing rats. J Nutr 125(9):2286-2293

Jobling M (2004) Are modifications in tissue fatty acid profiles following a change in diet the result of dilution? test of a simple dilution model. Aquaculture 232(1-4):551-562

Kanazawa A, Teshima SI, Ono K (1979) Relationship between essential fatty acid requirements of aquatic animals and the capacity for bioconversion of Linolenic acid to highly unsaturated fatty acids. Comp Biochem Physiol B 63(3):295-298

Kirsch PE, Iverson SJ, Bowen WD, Kerr SR, Ackman RG (1998) Dietary effects on the fatty acid signature of whole Atlantic cod (Gadus morhua). Can J Fish Aquat Sci 55(6):1378-1386

Knights B (1983) Food particle-size preferences and feeding-behaviour in warmwater aquaculture of European eel, Anguilla anguilla (L.). Aquaculture 30(1-4):173-190

Kuusipalo L, Kakela R (2000) Muscle fatty acids as indicators of niche and habitat in Malawian cichlids. Limnol Oceanogr 45(4):9961000

Lovern JA (1935) Fat metabolism in fishes - VI. The fats of some plankton crustacean. Biochem J 29:847-849

Makhutova ON, Kalachova GS, Gladyshev MI (2003) A comparison of the fatty acid composition of Gammarus lacustris and its food sources from a freshwater reservoir, Bugach, and the saline Lake Shira in Siberia, Russia. Aquat Ecol 37(2):159-167

Malzahn AM, Aberle N, Clemmesen C, Boersma M (2007) Nutrient limitation of primary producers affects planktivorous fish condition. Limnol Oceanogr 52(5):2062-2071

Malzahn AM, Hantzsche F, Schoo KL, Boersma M, Aberle N (2010) Differential effects of nutrient-limited primary production on primary, secondary or tertiary consumers. Oecologia 162(1):35-48

Mann RHK, Blackburn JH (1991) The biology of the eel Anguilla anguilla (L.) in an English chalk stream and interactions with juvenile trout Salmo trutta (L.) and salmon Salmo salar (L.). Hydrobiologia 218(1):65-76

Regost C, Arzel J, Cardinal M, Rosenlund G, Kaushik SJ (2003) Total replacement of fish oil by soybean or linseed oil with a return to fish oil in Turbot (Psetta maxima): 2. Flesh quality properties. Aquaculture 220(1-4):737-747

Reiser R, Stevenson B, Kayama M, Choudhury RBR, Hood DW (1963) The influence of dietary fatty acids and environmental 
temperature on the fatty acid composition of teleost fish. J Am Oil Chem Soc 40(10):507-513

Robin JH, Regost C, Arzel J, Kaushik SJ (2003) Fatty acid profile of fish following a change in dietary fatty acid source: model of fatty acid composition with a dilution hypothesis. Aquaculture 225 (1-4):283-293

Saito H, Murata M (1998) Origin of the monoene fats in the lipid of midwater fishes: relationship between the lipids of myctophids and those of their prey. Mar Ecol Prog Ser 168:21-33

Saito H, Ishihara K, Murase T (1997) The fatty acid composition in tuna (Bonito, Euthynnus pelamis) caught at three different localities from tropics to temperate. J Sci Food Agric 73(1):53-59

Saito H, Kotani Y, Keriko JM, Xue CH, Taki K, Ishihara K, Ueda T, Miyata S (2002) High levels of n-3 polyunsaturated fatty acids in Euphausia pacifica and its role as a source of Docosahexaenoic and Icosapentaenoic acids for higher trophic levels. Mar Chem 78(1):9-28

Sargent JR, Bell JG, Bell MV, Henderson RJ, Tocher DR (1995) Requirement criteria for essential fatty acids. J Appl Ichthyol 11(3-4):183-198

Schulze T, Kahl U, Radke RJ, Benndorf J (2004) Consumption, abundance and habitat use of Anguilla anguilla in a mesotrophic reservoir. J Fish Biol 65(6):1543-1562

Shiao JC, Lozys L, Iizuka Y, Tzeng WN (2006) Migratory patterns and contribution of stocking to the population of European eel in Lithuanian waters as indicated by otolith $\mathrm{Sr}$ : Ca ratios. J Fish Biol 69(3):749-769

Sinha V, Jones J, Pritchett R (1975) The European freshwater eel. Liverpool University Press, Liverpool
Steffens W (1997) Effects of variation in essential fatty acids in fish feeds on nutritive value of freshwater fish for humans. Aquaculture 151(1-4):97-119

Takeuchi T, Arai S, Watanabe T, Shimma Y (1980) Requirement of eel (Anguilla japonica) for essential fatty-acids. Bull Jpn Soc Sci Fish 46(3):345-353

Tesch FW (1999) Der Aal, 3rd edn. Paul Parey Verlag, Hamburg/ Berlin

Thibault I, Dodson JJ, Caron F (2007a) Yellow-stage American eel movements determined by microtagging and acoustic telemetry in the St Jean River watershed, Gaspe, Quebec, Canada. J Fish Biol 71(4): 1095-1112

Thibault I, Dodson JJ, Caron F, Tzeng WN, Iizuka Y, Shiao JC (2007b) Facultative catadromy in American eels: testing the conditional strategy hypothesis. Mar Ecol Prog Ser 344:219-229

Vanderploeg HA, Liebig JR, Gluck AA (1996) Evaluation of different phytoplankton for supporting development of Zebra Mussel larvae (Dreissena polymorpha): the importance of size and polyunsaturated fatty acid content. J Great Lakes Res 22(1):36-45

Vanvliet T, Katan MB (1990) Lower ratio of n-3 to n-6 fatty-acids in cultured than wild fish. Am J Clin Nutr 51(1):1-2

Vinson MR, Budy P (2011) Sources of variability and comparability between salmonid stomach contents and isotopic analyses: study design lessons and recommendations. Can J Fish Aquat Sci 68(1):137-151

Wickins JF (1985) Growth variability in individually confined elvers, Anguilla anguilla (L.). J Fish Biol 27(4):469-478

Zhukova NV, Aizdaicher NA (1995) Fatty acid composition of 15 species of marine microalgae. Phytochemistry 39(2):351-356 